\title{
Linx
}

Revue des linguistes de l'université Paris X Nanterre

$81 \mid 2020$

Enseignement des langues : la grammaire et la linguistique sont-elles solubles dans la pédagogie de projet?

\section{Pour une information grammaticale au service des usages et d'une dynamique de l'appropriation en L2}

Providing relevant grammatical information to support usages and appropriation processes in $\mathrm{L} 2$

\section{Catherine Felce}

\section{(2) OpenEdition}

\section{Journals}

Édition électronique

URL : http://journals.openedition.org/linx/7166

DOI : 10.4000/linx.7166

ISSN : 2118-9692

Éditeur

Presses universitaires de Paris Nanterre

\section{Édition imprimée}

Date de publication : 15 décembre 2020

ISSN : 0246-8743

Référence électronique

Catherine Felce, «Pour une information grammaticale au service des usages et d'une dynamique de l'appropriation en L2 », Linx [En ligne], 81 | 2020, mis en ligne le 15 décembre 2020, consulté le 29

janvier 2021. URL : http://journals.openedition.org/linx/7166 ; DOI : https://doi.org/10.4000/linx.7166

Ce document a été généré automatiquement le 29 janvier 2021.

Département de Sciences du langage, Université Paris Ouest 


\title{
Pour une information grammaticale au service des usages et d'une dynamique de l'appropriation en L2
}

Providing relevant grammatical information to support usages and appropriation processes in $\mathrm{L} 2$

\author{
Catherine Felce
}

\section{Introduction}

'Grammaire' et 'approche à visée actionnelle' - la juxtaposition des deux termes semble opposer d'entrée de jeu des aspects qui relèveraient pour l'un d'une description théorique du système linguistique d'une langue donnée et pour l'autre d'une méthodologie d'enseignement basée sur l'utilisation effective et sociale de cette même langue. Mais ce qui s'oppose ici, c'est également une vision normative et prescriptive orientée vers la correction linguistique et une perspective qui met l'accent sur les usages dans leur variété, souligne leur caractère situé et admet la variation comme réalité langagière inhérente à toute performance. Derrière l'opposition des termes se jouent cependant aussi des antagonismes plus profonds qui continuent de marquer non seulement le domaine de l'enseignement / apprentissage, mais aussi le champ de la didactique des langues. Il arrive ainsi d'opposer linguistes et didacticiens et d'invoquer le manque de grammaire (on comprendra de rigueur) pour critiquer l'approche actionnelle, ou ce que l'on considère comme ses dérives (la communication à n'importe quel prix). On peut reconnaître dans cette contradiction l'opposition entre théorie et pratique, c'est-à-dire entre une science plus 'dure', dont seraient issues les théories grammaticales, et des savoirs de nature plus praxéologique, au service de l'intervention pédagogique. À un niveau interne à l'apprenant enfin, une troisième opposition concerne la nature des connaissances devant faire l'objet d'un apprentissage en classe de langue. Si l'enseignement institutionnel prend désormais en compte la complexité des compétences en jeu dans les différentes activités langagières, le travail sur les 
formes de la langue tend encore à privilégier un savoir métalinguistique, de type déclaratif (des règles que l'on peut énoncer), alors que la capacité à communiquer de manière fluide et en temps réel reposerait sur des connaissances moins formalisées et relèverait, par conséquent, d'autres formes de mémorisation et d'apprentissage (Ellis, 2008 : 417-420).

2 Au début des années 80, Claire Kramsch (1983) publie un article dans lequel elle évoque la fonction communicative des règles de grammaire. Elle y défend une conception qui refuse de réduire la grammaire à un ensemble autonome de propriétés formelles et propose, à l'inverse, de comprendre et d'enseigner les formes en s'appuyant sur les fonctions qu'elles endossent dans le discours. Ses remarques n'ont aujourd'hui rien perdu de leur actualité quand nous posons la question de savoir comment aborder la grammaire dans une approche qui met en avant les usages de la langue apprise. Pour Rod Ellis, l'enseignement grammatical permet autant d'accéder à une compréhension métalinguistique des formes qu'à accompagner les apprenants dans un traitement adéquat de ces formes, en réception comme en production, et en permettre ainsi l'appropriation (Ellis, $2006: 84$ ). C'est dans cette conception que s'inscrit notre contribution.

3 À travers l'exemple de l'ordre des mots en allemand et de son enseignement à des apprenants débutants, nous montrerons dans un premier temps pourquoi les règles grammaticales positionnelles ne suffisent pas à décrire les options de linéarisation de l'énoncé de manière adéquate. Dans un deuxième temps, nous reformulerons les objectifs linguistiques pouvant être associés à la réalisation de l'ouverture de l'énoncé en allemand pour en faire des enjeux acquisitionnels, c'est-à-dire des étapes pour l'acquisition de formes spécifiques utiles à des usages identifiés. Nous proposerons enfin une progression linguistique qui intègre des aspects moins normés du fonctionnement langagier et tente de s'accorder au travail cognitif qui sous-tend l'apprentissage (Bange, 2005).

\section{Le placement verbal correct : un critère parmi d'autres}

4 D'un point de vue typologique, l'allemand fait partie des langues dites à verbe second, en référence à la position qu'occupe le verbe fléchi dans une déclarative, mais il est aussi courant de qualifier l'allemand de langue à verbe final. On se place pour cela dans la perspective du groupe verbal à l'infinitif au sein duquel les relations se font de la base verbale en remontant vers la gauche, à rebours de l'ordre progressif qui caractérise d'autres langues comme le français ou l'anglais. Une autre particularité est liée au fait qu'en allemand, le positionnement verbal second constitue une borne fixe dans l'énoncé qui limite l'occupation de la position préverbale à un seul élément et impose au locuteur d'opérer une sélection parmi une variété de candidats potentiels. En effet, le champ initial (Vorfeld) n'est pas une position syntaxiquement déterminée (Zifonun et al., 1997 : 1639), il peut au contraire être occupé par des constituants de nature et de fonction très diverses : le sujet, un groupe prépositionnel, un complément ou encore une subordonnée entière, dont la présence en position préverbale conditionne alors un positionnement du sujet après le verbe fléchi. Des constructions de type X-Verbe-Sujet ou encore Objet-Verbe-Sujet sont de ce fait courantes en allemand et ne sont pas sans poser problème à des apprenants francophones qui 
attribuent généralement la position préverbale au sujet de la phrase. Les préférences à l'ouverture ne se recouvrent donc pas nécessairement selon les langues; quand pour d'autres langues, c'est la fonction syntaxique qui est déterminante, ces préférences dépendent en allemand de critères informationnels, pragmatiques et textuels (Fandrych, 2005; Samson, 2004; Speyer, 2008) dont la grammaire scolaire ne rend pas nécessairement compte.

\subsection{La règle et ses limites}

5 La position du verbe fléchi dans la phrase détermine la modalité (assertive, interrogative, subordonnée) de la proposition. Le positionnement verbal second identifie un énoncé assertif, mais il est aussi présenté comme règle aux apprenants pour la construction de la phrase correcte, sans pour autant leur fournir les indications nécessaires pour la réalisation de l'ouverture. Tout se passe comme si l'hétérogénéité des expressions précédant le verbe était à rapporter à une absence de critères ou laissée à la liberté de choix du locuteur, alors que l'occupation du champ initial est contrainte par les différents paramètres de la situation de communication et que ce champ constitue une place stratégique (Marschall, 2012) à plusieurs titres. Il participe de la répartition et de la hiérarchisation de l'information entre des contenus informationnels connus, donnés ou déjà activés (le thème, le topique) et des unités apportant une information nouvelle, non encore présente dans le savoir partagé des interlocuteurs (le rhème, le commentaire ou le focus). Le dynamisme de la proposition (Schanen \& Confais, 2005 : 584-590) dépend des options de linéarisation, neutre dans le cas d'une progression du connu vers le nouveau (1) ou au contraire expressive ou marquée quand des éléments rhématiques porteurs d'un accent contrastif sont déplacés vers l'avant (2).

(1) Morgen fahre ich nach Paris.

[Demain, je vais à Paris.]

Ich fahre morgen nach Paris.

[Je vais demain à Paris.]

(2) ${ }^{+}$Arbeiten sollst du!

[Travailler, c'est ça que tu dois faire !]

Le Vorfeld accueille également en priorité des expressions cadratives qui assurent l'ancrage situatif ou contextuel des énoncés (3); il est aussi exploité au service de la mise en place d'une succession d'intervalles (temporels ou spatiaux) établissant un cadre référentiel (von Stutterheim, 1997) pour le déploiement informationnel au sein d'un récit chronologique ou d'une description spatiale (4).

(3) Im Jahr 2018 wollten 74 Prozent der Deutschen in den Sommermonaten verreisen.

[En 2018, 74\% des allemands voulaient partir en voyage pendant les mois d'été.]

(4) Mein Bett, das steht schräg ins Zimmer rein, und darüber habe ich mir so ein Himmelbett gemacht, selber gebaut. Das ist sehr gemütlich. Rechts neben dem Bett ist ein kleines Kästchen mit ganz vielen Schubladen und Sachen drin. Und darauf ist eine Lampe, (...)

[Mon lit, il est en diagonale dans la chambre, et au-dessus, je me suis fait un baldaquin, que j'ai construit moi-même. C'est très confortable. À droite du lit, il y a une petite boîte avec beaucoup de tiroirs et d'objets. Et dessus, il y a une lampe, (...)] 
7 Enfin, c'est à l'ouverture que se réalise la cohésion inter-phrastique (5); à travers la reprise initiale d'un contenu propositionnel activé en amont, une continuité de sens entre les énoncés est ainsi explicitement marquée (Dalmas, 2005 : 108).

(5) Meine Lieblingsfächer sind Englisch, Musik und Geschichte. (...). Geschichte mag ich, weil (...)

[Mes matières préférées sont l'anglais, la musique et l'histoire. (...). L'histoire j'aime ça, parce (...)]

8 Il n'y a donc ni hasard, ni totale liberté dans la sélection de l'élément qui ouvre l'énoncé. Les constituants susceptibles d'occuper le champ initial sont en concurrence et la sélection repose sur des aspects informationnels ou textuels qui ne sont pas formalisés dans des règles prescriptives et que l'enseignement grammatical laisse alors de côté.

9 La focalisation sur le placement verbal et la perspective essentiellement phrastique sont problématiques à plusieurs égards. Ils se réfèrent à un système figé et n'intègrent pas la variabilité des usages et des situations communicatives. Ils font également peu de cas du coût cognitif que représente un traitement basé sur la mobilisation d'un savoir déclaratif pour la production verbale (Skehan \& Foster, 2001), de surcroit en début d'apprentissage. Celle-ci exige d'affecter les ressources attentionnelles à ce seul traitement, aux dépens de celui du sens ou du contexte, alors que c'est bien sur le traitement du sens en profondeur que reposent des apprentissages durables (NarcyCombes, 2005 : 35). Enfin, la règle du placement verbal considère l'énoncé de manière isolée et non pas en lien avec ceux qui le précèdent ou lui succèdent ; elle ignore par conséquent la réalité du texte comme un tout localement planifié et structuré. Les phrases peuvent dès lors être correctes sur le plan syntaxique, sans pour autant être recevables au regard de leur environnement textuel ou de la situation communicative donnée.

10 Ainsi, dans des productions d'apprenants de différentes langues sources (suédois, anglais, français, chinois) attestant parfois pourtant d'une parfaite maîtrise morphosyntaxique, plusieurs études (Bohnacker \& Rosén, 2008; Breckle \& Zinsmeister, 2010; Dalmas, 2005; Lipsky, 2010) ont révélé des écarts entre les énoncés en L2 et ceux de locuteurs natifs. Il est intéressant de voir que c'est à l'ouverture que se cristallisent les difficultés : suroccupation du champ initial, préférence pour le sujet à l'ouverture au détriment d'expressions cadratives, manque de cohésion interphrastique dû à la méconnaissance de la fonction du champ initial pour l'établissement de la cohésion. Les préférences des langues sources observées demeurent opératoires en dépit d'un niveau de compétence avancé (von Stutterheim et al., 2002) et les connaissances morphosyntaxiques ne suffisent pas à leur recomposition pour un traitement langagier adéquat en L2.

11 À la suite de Dalmas $(2005,2013)$, nous postulons que des explications grammaticales centrées sur la construction de la phrase correcte n'apporte aux apprenants ni l'information linguistique pertinente pour comprendre les enjeux de l'ouverture, ni ne leur propose des activités visant à favoriser l'appropriation de compétences discursives. Dalmas relève que ceux-ci ne sont non seulement pas en mesure de produire des textes qui correspondent aux critères de textualité attendus, mais qu'ils décodent également de manière erronée des énoncés en langue cible : le déplacement d'un constituant vers l'avant étant systématiquement interprété comme mise en relief 
là où la topicalisation est utilisée pour retarder l'apparition de l'élément auquel on souhaite conférer un poids informationnel plus grand.

(6) Meine Frau habe ich im Kino kennen gelernt.

[Ma femme, je l'ai rencontrée au cinéma.]

Dans l'exemple (6), cité par Dalmas (2005, p. 102), il n'y a aucune raison d'interpréter le groupe nominal meine Frau comme étant mis en relief et traduit en français par une construction clivée (c'est... que ...) si celui-ci ne porte pas d'accent contrastif. Dans ce cas, il relève du thème, d'un contenu informationnel déjà mentionné, et c'est le lieu de la rencontre (im Kino) qui constitue alors l'information apportée. La mise en relief n'est pertinente que si le groupe nominal initial est accentué et est à interpréter comme contenu rhématique.

\subsection{Pour une prise en compte d'aspects moins normés}

Bien avant l'adoption de l'approche actionnelle, Kramsch (1983) évoque la nécessité de regarder le fonctionnement syntaxique de l'allemand à la lumière de principes discursifs. Constatant chez des apprenants anglophones une tendance à construire des énoncés conformément aux principes de leur L1, c'est-à-dire en privilégiant le sujet en position initiale, elle souligne la nécessité d'enseigner les savoir-faire discursifs spécifiques de l'allemand en privilégiant le recours à des notions pragmaticodiscursives (thème, topique) par rapport aux traditionnelles catégories morphosyntaxiques (sujet). Dans l'article que nous mentionnons, paru il y a une quinzaine d'années déjà, Dalmas plaide pour que l'enseignement accorde moins d'importance aux conventions et dote les apprenants des moyens de s'approprier le rôle stratégique de l'ouverture pour la production et la réception conforme des discours/textes en allemand (Dalmas, 2005 : 97). Face au manque d'habileté discursive des apprenants, elle préconise un enseignement des principes textuels et informationnels propres à l'allemand. Fandrych $(2003: 174)$ ajoute quant à lui que la réflexion sur les possibilités d'occupation du Vorfeld devrait dès le début être intégrée aux apprentissages. On retrouve chez tous ces chercheurs des préconisations qui sont également formulées dans le Cadre Européen Commun de Référence pour les Langues (CECRL) quant à la nécessité de développer au sein de la composante linguistique une compétence discursive et textuelle qui permette la production et l'ordonnancement de phrases au sein de textes cohérents. Cette dernière recouvre entre autres :

- la connaissance de l'organisation des phrases et de leurs composantes

- la capacité à les maîtriser en termes

- de thème/rhème

- d'information donnée/information nouvelle [...]

- la capacité de gérer et de structurer le discours en termes

- d'organisation thématique

- de cohérence et de cohésion

- d'organisation logique [...]

(Conseil de l'Europe, 2001 : 96)

14 Ces paramètres et ces connaissances linguistiques peinent pourtant à pénétrer les pratiques et les descriptions grammaticales scolaires. On peut y voir la tendance à considérer les principes textuels et organisationnels des discours comme des universaux et à oublier qu'ils correspondent à des préférences différentes ou adoptent 
des formes spécifiques selon la structure des langues. Mais surtout, les aspects évoqués s'éloignent de la grammaire de la phrase et de l'aspect stable et rassurant que peuvent avoir des règles univoques et simples à formuler. Ainsi, le savoir grammatical dont disposent les apprenants pour construire leurs énoncés en allemand se limite-t-il aux règles qui s'appliquent aux positions syntaxiques fixes; les règles moins strictement normées, en relation avec les usages et la production de discours/textes sont quant à elles ignorées.

\section{Enseignement grammatical et tâches de production}

Comment dès lors concevoir une information grammaticale pertinente pour les apprenants dans le cadre d'une approche mettant en avant les usages de la langue cible ? Il s'agit de les amener à développer des connaissances et des procédures adéquates pour le traitement de L2 et de favoriser à travers les tâches l'appropriation des formes correspondant aux préférences organisationnelles de l'allemand (au niveau syntagmatique, informationnel et textuel). Les pistes que nous envisageons s'appuient à la fois sur une compréhension linguistique des phénomènes de linéarisation et sur l'analyse de productions d'apprenants recueillies à partir de tâches effectuées en classe (Felce, 2015).

Ces productions ont été recueillies auprès d'un groupe de 24 élèves ( 7 garçons et 17 filles entre 11 et 13 ans) scolarisés dans un établissement secondaire d'île-de-France, au fil de leurs deux premières années d'apprentissage de la langue au collège (soit en classe de $6^{\text {ème }}$ et en $5^{\text {ème}}$ ).

Tableau 1 : Tableau synthétique des tâches mentionnées

\begin{tabular}{|c|c|c|}
\hline Intitulé & Caractéristiques et enjeux & $\begin{array}{l}\text { Nombre d'énoncés } \\
\text { pris en compte : } N=\end{array}$ \\
\hline $\begin{array}{l}\text { wochenplan } \\
\text { Interaction orale avec un } \\
\text { partenaire } \\
\text { Objectif communicatif : } \\
\text { Reconstituer le planning de } \\
\text { la semaine de son partenaire }\end{array}$ & $\begin{array}{l}\text { - Usage des groupes verbaux d'activités appris } \\
\text { - } \quad \text { Schéma interactif question / réponse } \\
\text { - } \quad \text { Alternance à l'ouverture guidé par la } \\
\text { question } \\
\text { - Mémorisation d'associations Verbe-Sujet }\end{array}$ & 183 \\
\hline $\begin{array}{l}\text { kino } \\
\text { Évaluation de l'interaction } \\
\text { orale avec un partenaire } \\
\text { Objectif communicatif : } \\
\text { Trouver un créneau commun } \\
\text { pour aller au cinéma }\end{array}$ & $\begin{array}{l}\text { - Échange d'informations en fonction des } \\
\text { emplois du temps dont chacun dispose } \\
\text { - } \quad \text { Variations possibles du schéma interactif } \\
\text { - } \quad \text { Plus grande variété de bases verbales } \\
\text { - } \quad \text { Utilisation de da pour éviter la répétition }\end{array}$ & 154 \\
\hline $\begin{array}{l}\text { tagesablauf: } \\
\text { Évaluation de la production } \\
\text { écrite } \\
\text { Objectif communicatif : } \\
\text { Écrire un article qui décrit le } \\
\text { quotidien de jeunes } \\
\text { adolescents étrangers }\end{array}$ & $\begin{array}{l}\text { - Un document support précisant horaires et } \\
\text { événements associés pour différents } \\
\text { adolescents } \\
\text { - Mise en place du décalage temporel } \\
\text { (expressions employées) } \\
\text { - Similitude de dann et danach (rappel de la } \\
\text { fonction de da) } \\
\text { - Cohésion inter-phrastique }\end{array}$ & 305 \\
\hline $\begin{array}{l}\text { meine_klasse: } \\
\text { Évaluation de la production } \\
\text { écrite } \\
\text { Objectif communicatif : } \\
\text { Présenter sa classe à une } \\
\text { classe partenaire à partir } \\
\text { d'une photo }\end{array}$ & $\begin{array}{l}\text { - Utilisation des prépositions spatiales sans } \\
\text { nécessité de réfléchir à la morphologie } \\
\text { nominale (prénoms) } \\
\text { - Décalage spatial sur le modèle du décalage } \\
\text { temporel } \\
\text { - Recours à un schéma conceptuel similaire en } \\
\text { L1 pour favoriser le positionnement verbal }\end{array}$ & 181 \\
\hline $\begin{array}{l}\text { zimmer: } \\
\text { Évaluation de la production } \\
\text { écrite } \\
\text { Objectif communicatif : } \\
\text { Décrire sa chambre à son } \\
\text { correspondant }\end{array}$ & $\begin{array}{l}\text { - Utilisation des prépositions spatiales } \\
\text { (+morphologie nominale spécifique en cours } \\
\text { d'acquisition) } \\
\text { - } \quad \text { Mise en place de différents intervalles } \\
\text { spatiaux } \\
\text { - } \quad \text { Formation d'expressions } d a+\text { préposition. }\end{array}$ & 240 \\
\hline
\end{tabular}



communicatives proposées en classe de langue en fonction du niveau compétence des apprenants (A1/A2). Elles ont été réalisées sur le temps de classe, en temps limité et sans recours à des aides.

Elles sont à voir comme des étapes dans une progression d'apprentissage. Il s'agit en premier lieu d'amener les apprenants à hiérarchiser les contenus propositionnels dans les énoncés de telle sorte que cette mise en ordre corresponde à une progression informationnelle pertinente selon le contexte, les intentions communicatives du locuteur ou l'appréciation de l'état de savoir de l'interlocuteur. Un deuxième aspect consiste à aborder dès le début des principes qui régissent la cohésion inter-énoncés et à favoriser l'appropriation de formes spécifiques à l'établissement de la textualité particulière de l'allemand.

\subsection{Une approche implicite de la grammaire}

19 La production langagière repose sur deux types de connaissances et de stockage mémoriel distincts. Les locuteurs d'une langue peuvent appliquer des règles (rule-based system) dans un processus analytique, ou bien recourir à un fonctionnement lexical en rappelant des constructions toutes faites et mémorisées telles quelles (exemplar-based system) (Skehan, 1998; Skehan \& Foster, 2001). La différence réside dans le coût attentionnel associé à chacun de ces processus. Si le traitement langagier à partir de règles est plus exigeant et laborieux, les blocs lexicalisés (chunks) sont mobilisables sans surcoût cognitif. Stockées sous forme d'items lexicaux, ces constructions sont plus aisément rappelées quand il s'agit de répondre aux besoins immédiats de la communication et à l'exigence de fluidité et d'efficacité. Par ailleurs, leur usage répété, voire ritualisé, renforce la fixation mémorielle des associations qu'elles mettent en jeu. Un premier palier de l'apprentissage linguistique intervient dès la découverte des groupes verbaux et de leur structure particulière :

(7) Tischtennis spielen - jouer au ping-pong

Tanzkurs haben - avoir cours de danse

schwimmen gehen - aller nager

Deutsch lernen - apprendre l'allemand

21 De nombreux groupes verbaux peuvent être mémorisés par le biais de différentes activités, en faisant des aller-retours avec le français de manière à souligner l'ordre spécifique des constituants. Ce travail initial peut donner lieu à des tâches sous forme de jeux ou encore de listes ordonnées : classer les activités selon qu'on les pratique ou pas, qu'on les apprécie ou pas, les devoirs scolaires que l'on fait tout seul ou en groupe, les activités quotidiennes que différents membres de la famille effectuent, etc. Ce faisant, c'est bien du sens qui est traité en priorité, les formes ne l'étant que de manière incidente à travers leur manipulation et leur répétition.

Dans un second temps, il est possible de familiariser les apprenants avec des tournures courantes qui commencent par un autre constituant de la phrase que le sujet. De nombreux rituels de classe (tableau 1) offrent cette possibilité et il est aisé de les exploiter très tôt avec les apprenants. Ils serviront ultérieurement de réalisations exemplaires quand il s'agira d'introduire une réflexion métalinguistique sur la construction des énoncés. 
Tableau 2 : Exemples de rituels de classe usant d'expressions cadratives à l'ouverture

\begin{tabular}{|c|c|}
\hline Domaine & \multicolumn{1}{c|}{ Exemples d'énoncés } \\
\hline date & $\begin{array}{l}\text { Heute haben wir Donnerstag, den ... [Aujourd'hui, nous sommes jeudi, } \\
\text { le... } \\
\text { Heute ist ... [Aujourd'hui c'est...] }\end{array}$ \\
\hline appel & $\begin{array}{l}\text { Heute ist X nicht da. [Aujourd'hui, X n'est pas là.] } \\
\text { Heute fehlt niemand. [Aujourd'hui, il ne manque personne.] } \\
\text { In der Klasse sind wir 28. [Dans la classe, nous sommes 28.] }\end{array}$ \\
\hline météo & $\begin{array}{l}\text { Heute Morgen scheint die Sonne. [Ce matin, le soleil brille.] } \\
\text { Heute Nachmittag regnet es. [Cet après-midi, il pleut.] }\end{array}$ \\
\hline santé & $\begin{array}{l}\text { Heute geht's mir gut. [Aujourd'hui ça va bien.] } \\
\text { Mir geht's gut. [Moi, ça va bien.] }\end{array}$ \\
\hline devoirs & $\begin{array}{l}\text { In Deutsch haben wir viel auf. [En allemand, on a plein de devoirs.] } \\
\text { In Mathe gibt der Lehrer nicht viele Hausaufgaben. [En maths, le prof } \\
\text { ne donne pas beaucoup de devoirs.] } \\
\text { Für Montag sollen wir die Übung machen. [Pour lundi, nous devons } \\
\text { faire l'exercice.] }\end{array}$ \\
\hline événements & $\begin{array}{l}\text { Heute hat X Geburtstag. [Aujourd'hui, c'est l'anniversaire de X.] } \\
\text { Morgen bin ich nicht da. [Demain, je ne suis pas là.] } \\
\text { Morgen haben wir keine Schule. [Demain, on n'a pas cours.] }\end{array}$ \\
\hline rappels à l'ordre & $\begin{array}{l}\text { Jetzt seid ihr bitte still! [Maintenant silence SVP !] } \\
\text { Jetzt ist Schluss! [Maintenant ça suffit !] } \\
\text { Jetzt sollt ihr gut zuhören! [Maintenant vous devez être attentifs !] }\end{array}$ \\
\hline repas & $\begin{array}{l}\text { In der Kantine essen wir heute Fisch. [À la cantine, on mange du } \\
\text { poisson aujourd'hui.] } \\
\text { Heute gibt's Eis. [Aujourd'hui, il y a des glaces.] }\end{array}$ \\
\hline
\end{tabular}

23 Il n'est pas encore nécessaire à ce stade d'apporter de commentaires métalinguistiques au sujet du placement verbal; les élèves saisissent d'eux-mêmes la pertinence de la spécification posée à l'ouverture et le cadre de validité qu'elle établit. Heute [aujourd'hui] sert par exemple à circonscrire les contenus propositionnels à l'intérieur d'un périmètre précis : l'absence d'un élève, l'anniversaire d'un autre ou le temps qu'il fait, valent pour aujourd'hui et pas dans l'absolu.

Ces formules toutes faites, répétées de nombreuses fois, ont plusieurs vertus : elles contribuent à familiariser les apprenants à la fonction cadrative assurée par l'élément positionné dans le Vorfeld; leur répétition renforce la mise en mémoire d'associations Verbe-Sujet qui pourront être réactivées dans d'autres contextes ; elles banalisent aussi des usages tout en évitant de focaliser l'attention sur des aspects grammaticaux (positionnement verbal) contraignants par rapport aux préférences de la langue source.

Il est par ailleurs possible de mettre à profit la disponibilité de ces constructions XVerbe mémorisées pour introduire des formes spécifiques, courantes en allemand et garantes d'une continuité de sens entre énoncés successifs. L'introduction de l'expression pronominale das [ça] et de l'élément plurifonctionnel da [là, dans ce domaine-là, sur ce point-là, à ce moment-là, etc.] constitue en effet un premier jalon vers l'usage de moyens cohésifs. 
Tableau 3 : Exemples de tournures préparant à l'usage de premières expressions anaphoriques

\begin{tabular}{|c|c|}
\hline $\begin{array}{c}\text { communication de } \\
\text { classe }\end{array}$ & $\begin{array}{c}\text { (une question est posée) - réaction : Das weiß ich nicht. [Ça, je n'en } \\
\text { sais rien.] } \\
\text { (quelque chose est dit) - réaction : Das verstehe ich nicht. [Je ne } \\
\text { comprends pas ce que vous venez de dire] }\end{array}$ \\
\hline les goûts & $\begin{array}{c}\text { Sport, das mag ich nicht. [Le sport, ça j'adore] } \\
\text { Freunde treffen, das mag ich sehr. [Retrouver des amis, ça ça me } \\
\text { plaît.] } \\
\text { Geschichte, das finde ich total interessant. [L'histoire, je trouve ça } \\
\text { super intéressant.] } \\
\text { Grammatikübungen machen, das finde ich blöd. [Faire des exercices } \\
\text { de grammaire, je trouve ça stupide.] }\end{array}$ \\
\hline les résultats & $\begin{array}{c}\text { Ich bin sehr gut in Deutsch, da habe ich eine Eins. [Je suis très bon } \\
\text { en allemand, dans cette matière-là j'ai 20.] }\end{array}$ \\
\hline l'emploi du temps & $\begin{array}{c}\text { Hast du Zeit am Montag? Nein, da habe ich Sport! [Tu as du temps } \\
\text { lundi? Non, ce jour-là j'ai sport.] }\end{array}$ \\
\hline
\end{tabular}

À travers des formulations telles que celles mentionnées dans le tableau (3), les apprenants ont l'occasion de manipuler des éléments pronominaux qui ont un rôle essentiel en allemand pour établir des liens anaphoriques et construire par leur truchement une continuité propositionnelle spécifique à la langue. Là encore, ces expressions sont utilisées et répétées sans recours à des explications grammaticales ; on peut simplement laisser les apprenants formuler les similitudes et les différences qu'ils repèrent entre le sémantisme de das [ça] et da [là, dans ce domaine-là, sur ce point-là, à ce moment-là, etc.]. En privilégiant une entrée via des tournures courantes, il s'agit de doter les apprenants de formes et de fonctions qu'il sera possible de rappeler dans le développement ultérieur de schémas plus complexes, mais cependant similaires.

\subsection{Hiérarchisation informationnelle des constituants de l'énoncé}

Une tâche couramment proposée aux apprenants débutants consiste à échanger sur les activités pratiquées dans la semaine, pour comparer leurs emplois du temps ou convenir d'un rendez-vous. Les élèves ont acquis en amont des groupes verbaux variés relatifs aux activités scolaires ou extrascolaires, ils savent préciser les jours de la semaine, les horaires et sont capables de formuler des questions. Nous avons décidé d'exploiter ici des schémas simples d'interaction, dans lesquels chaque tour de parole est constitué d'une question et de la réponse apportée. Deux types de questions sont possibles et vont susciter des réponses mettant en jeu deux organisations distinctes des constituants dans l'énoncé selon la distribution des rôles de topique et focus.

(8) Was hast / machst du am Montag? - Am Montag habe ich Theaterklub.

[Qu'est-ce que tu as / fais le lundi ? - Le lundi, j'ai club théâtre.]

Wann hast du Fußball? - Fußball ist am Mittwoch.

[Quand est-ce que tu as foot ? - Le foot, c'est le mercredi.]

L'exemple (8) illustre l'alternance entre une ouverture par une expression temporelle ou par la mention de l'activité, conformément à une progression informationnelle du connu/donné vers le nouveau. Ce qui est intéressant pour le traitement langagier ici, c'est que la réponse est guidée et, de fait, facilitée par la question. À travers le phénomène de reprise, explicitement encouragé dans l'activité, le locuteur dispose 
d'une ressource pour amorcer son tour de parole et réaliser dans le même temps la cohésion inter-discursive que la reprise prend en charge (Pochon-Berger, 2008).

(9) (MARN_CLAR_wochenplan)

150. CLAR: [...] Was machst du am Dienstag

[Qu'est-ce que tu fais le mardi ?]

151. MARN: Am Dienstag um 6 Uhr habe ich Musikkurs. Und du wann (was) hast du am

Dienstag?

[Le mardi à 6 heures, j'ai cours de musique. Et toi, tu fais quoi le mardi ?]

152. CLAR: Am Dienstag um 3 Uhr habe ich Schulorchester und um 6 Uhr spiele ich

Tischtennis.

[Le mardi à 3 heures, j'ai orchestre et à 6 heures, je joue au tennis.]

153. MARN: Wann ist Schulorchester?

[C'est quand l'orchestre?]

154. CLAR: Schulorchester ist am Dienstag um 3 Uhr.

[L'orchestre, c'est le mardi à 3 heures.]

On peut par ailleurs observer un taux de réussite important dans le positionnement verbal second et l'usage spontané des associations de type Verbe-Sujet que le caractère répétitif des échanges, au même titre que le phénomène de reprise, peut en partie expliquer.

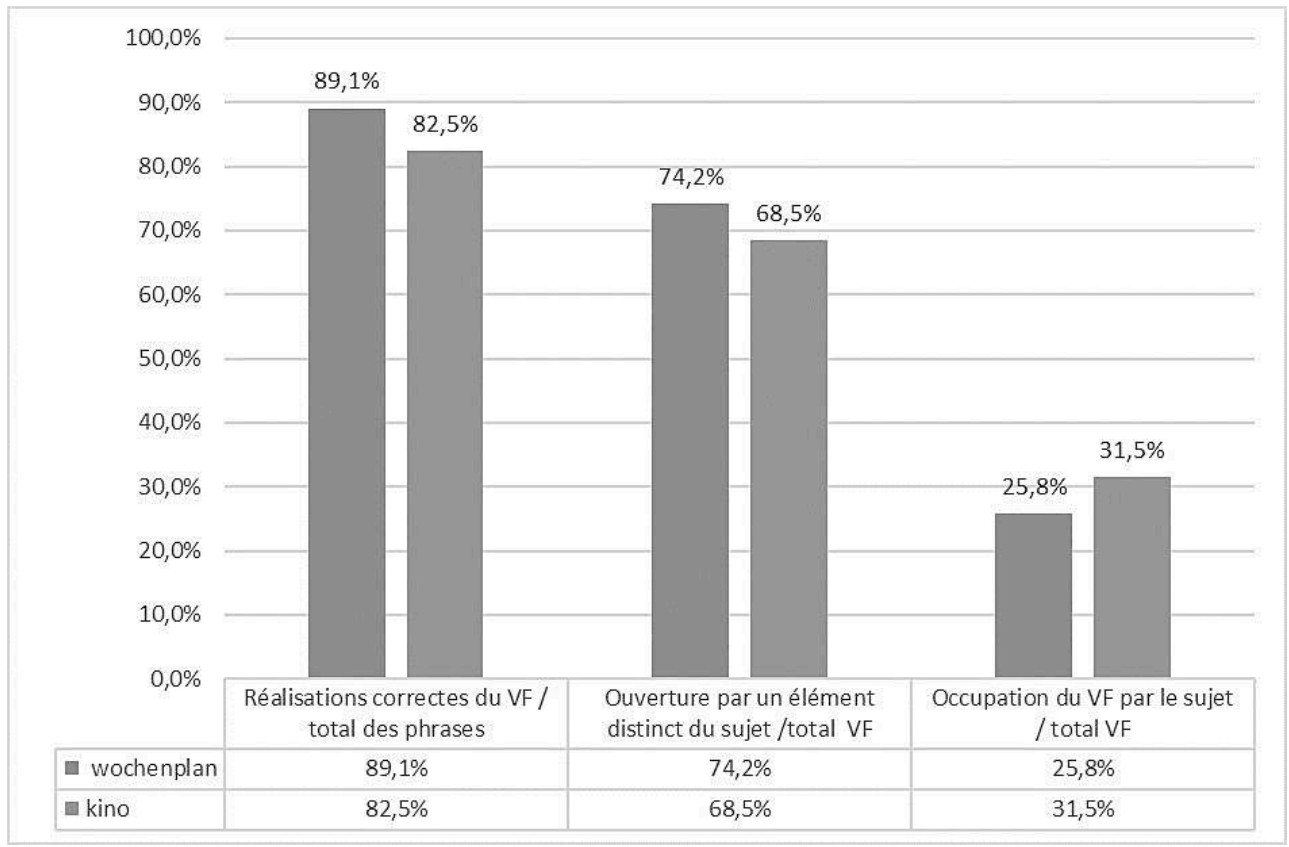

Graphique 1 : Réalisations correctes de l'ouverture pour les tâches interactives

Un tel procédé de reprise semble par ailleurs permettre l'émergence de constructions bien plus spécifiques, de type Objet-Verbe-Sujet, sans qu'il n'y ait eu de préparation ou d'explication grammaticale au préalable.

(10) (MEL_MAR_wochenplan)

139. MARI: Am Dienstag um 3 Uhr habe ich / spiele ich Flöte und am (um) 5 habe ich Schulorchester. Wann ist / wann ist Florettfechten?

Le mardi à 3 heures j'ai / je joue de la flûte et à 5 heures j'ai orchestre. C'est quand

le fleuret ?]

140. MEL: Florettfechten spiele (mache) ich am Mittwoch um 4 Uhr. Wann ist Klavier?

[Le fleuret, j'en fais le mercredi à 4 heures. Le piano, c'est quand ?]

31 L'ordre Objet-Verbe-Sujet est favorisé en (10) par la répétition du schéma interactif récurrent que nous avons évoqué. Le topique repris à l'ouverture se substitue aux 
catégories de sujet et d'objet que les apprenants peinent souvent à identifier dans la phrase et facilite de la sorte une structuration tout à fait spécifique à la langue cible. Le fait de recourir à des notions informationnelles semblerait contribuer à rendre une structure accessible, alors que son traitement syntaxique relèverait d'un stade plus avancé.

\subsection{Continuité temporelle et spatiale}

Au cours de la deuxième année d'apprentissage, l'élaboration de textes plus construits se met en place. La chronologie temporelle et la localisation dans l'espace sont fréquemment convoquées pour organiser des descriptions simples de l'environnement immédiat et personnel des apprenants.

Christiane von Stutterheim et ses collègues (Carroll \& Lambert, 2003, 2006; Lambert et al., 2008) se sont intéressées à l'organisation des récits en L1 et L2 et ont mis en évidence que le déploiement de l'information ne s'opérait pas selon les langues sources de la même façon. Ainsi, la mise en forme chronologique d'une succession d'actions s'inscrit-elle en allemand dans un mouvement référentiel opéré par un décalage temporel progressif, composé d'intervalles qui s'enchainent les uns à partir des autres, comme l'illustre l'exemple (11). En ce qui concerne la localisation d'objets dans un espace identifié, là encore, la progression se fait de proche en proche, chaque objet localisé servant à son tour de point de repère pour le suivant (voir l'exemple 4). Von Stutterheim parle de décalage (Verschiebung) pour caractériser ce mouvement qui a ceci de particulier que les intervalles temporels ou spatiaux posés se succèdent en se rattachant de manière explicite à un point repéré en amont (von Stutterheim, 1997 : 63-64).

Le travail en classe peut commencer à partir de textes exemplaires produits par des locuteurs germanophones. Les apprenants procèdent tout d'abord au repérage des moyens de construire cette succession d'intervalles, comme dans l'extrait (11):

(11) Nach der Schule laufe ich nach Hause und Esse zum Beispiel Kartoffeln mit Fleisch.

Danach mach ich sofort die Hausaufgaben am Esstisch. Nach den Hausaufgaben muss ich zur Ensembleprobe. Dort spielen wir dann auf der Posaune und andere auf der Tuba. Wenn wir fertig gespielt haben, laufe ich nach Hause mit der Posaune.

[Après l'école, je rentre à pied à la maison et je mange par ex. des pommes de terre et de la viande. Après ça je fais tout de suite mes devoirs sur la table à manger. Après les devoirs je dois aller aux répétitions de l'ensemble. Là-bas on joue alors du trombone et d'autres jouent du tuba. Quand on a fini de jouer, je rentre à la maison avec le trombone.]

Le repérage du décalage temporel opéré est d'autant plus facilité que les élèves l'observent à l'attaque de l'énoncé, à travers des formes qu'ils (re)connaissent : la préposition nach [après] a été manipulée pour l'expression de l'heure ou reconnue en décomposant le mot Nachmittag [après-midi]. Mais les élèves sont aussi capables d'identifier et de relier de nouvelles formes (danach [après ça]) à des usages déjà en place ou d'en produire d'autres par analogie (davor [avant ça]). En effet, le rappel de da et de la fonction de reprise d'un contenu informationnel précité qui lui est associé aide certainement à la compréhension et l'appropriation de complexes tels que danach $(d a+n a c h)$ ou davor (da+vor). Quant à dann [ensuite], son rapprochement avec da et danach tombe sous le sens. 
Les productions correspondant à la tâche tagesablauf témoignent d'une grande variété dans les expressions temporelles employées (tableau 4) et cette variété s'accompagne par ailleurs, là encore, d'un taux de réussite important dans le positionnement verbal second (86,9\% des énoncés sont corrects, avec $53,4 \%$ de constituants autres que le sujet à l'ouverture) et en dépit de la complexité (ou longueur) de certaines expressions choisies.

Tableau 4 : Variété des expressions temporelles pour la tâche tagesablauf

\begin{tabular}{|c|c|}
\hline Groupes prépositionnels & $\begin{array}{l}\text { um halb acht [à sept heures et demie] } \\
\text { von siebzehn Uhr bis etwa achtzehn Uhr [de } 17 \text { jusqu'à } \\
\text { environ } 18 \text { heures] } \\
\text { nach dem Frühstück [après le petit-déjeuner] } \\
\text { nach dem Essen [après le repas] } \\
\text { nach den Hausaufgaben [après les devoirs] } \\
\text { nach dem Basketball spielen [après avoir joué au basket] } \\
\text { nach (dem) Klavier üben [après les exercices au piano] } \\
\text { nach der (dem) Unterricht [après les cours] } \\
\text { nach der (dem) Schulschluss [après la fin des cours] }\end{array}$ \\
\hline Groupes adverbiaux & zuerst [d'abord] - dann [ensuite] - morgens [le matin] \\
\hline $\begin{array}{l}\text { Groupes nominaux avec } \\
\text { expansion }\end{array}$ & $\begin{array}{l}\text { eine Stunde nach dem Essen [une heure après manger] } \\
\text { etwa } 45 \text { Minuten später [environ } 45 \text { minutes plus tard] } \\
\text { ungefähr eine halbe Stunde später [à peu près une demi-heure } \\
\text { plus tard] } \\
\text { (Eine)Viertelstunde später [un quart d'heure plus tard] }\end{array}$ \\
\hline Subordonnées & $\begin{array}{l}\text { wenn die Schule aus ist [quand l'école est finie] } \\
\text { before (bevor) sie ins Bett geht [avant qu'elle aille se coucher] }\end{array}$ \\
\hline Expressions complexes & $\begin{array}{l}\text { dann, um sechs Uhr [ensuite à six heures] } \\
\text { nach dem Abendessen, wenn sie ihre Freundin an nicht ruft } \\
\quad \text { (nicht anruft) [après le repas, si elle ne téléphone pas à ses } \\
\text { amies] } \\
\text { erst dann [c'est seulement ensuite que] }\end{array}$ \\
\hline Formes idiosyncrasiques & $\begin{array}{l}\text { nach ihre Freunde treffen [après avoir retrouvé ses amis] } \\
\text { vor dem Freunde treffe [avant de retrouver les amis] } \\
\text { drei Stunden früher (vor dem) schlafen gehen [trois heures avant } \\
\text { d'aller se coucher] } \\
\text { nach (danach) [après (adverbial)] } \\
\text { eine Stunde nach (danach) [une heure après (adverbial)] }\end{array}$ \\
\hline
\end{tabular}

37 Ce qui est particulièrement intéressant dans l'exemple (12), c'est l'usage que l'apprenante fait de l'ouverture, à la fois pour imprimer la progression temporelle dans son texte, mais également pour assurer le raccord entre les énoncés successifs. La fonction des expressions anaphoriques formées à partir de $d a$, (avec une orientation vers l'aval et vers l'amont) est exploitée à bon escient, et on constate également que leur usage s'accompagne d'un positionnement verbal correct. 
(12) (IFI tagesablauf)

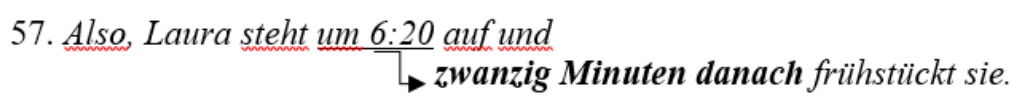

[Donc, Laura se lève à $6 \mathrm{~h} 20$ et $\mathbf{2 0}$ minutes après elle prend son petit déjeuner.]

(...) um eins isst sie zu Mittag.

$\longrightarrow$ Eine Stunde danach macht sie Hausaufgaben...

[(...) à une heure elle a le repas de midi. Une heure plus tard, elle fait ses devoirs...]

und spielt sie Klavier bis um fünf.

$\longrightarrow$ da trefft (trifft) sie Freunde...

[...et elle joue du piano jusqu'à 5 heures. Là elle retrouve des amis...]

...aber um sechs soll sie zu Hause sein.

$\longrightarrow$ Da isst sie nämlich zu Abend,

$\longrightarrow$ dann ruft sie Freundin(nen) an (...)

[...mais à 6 heures elle doit être à la maison. C'est à cette heure en effet qu'elle mange le repas du soir, ensuite elle appelle des amies (...)]

Figure 1 : Moyens mis en œuvre pour établir le décalage temporel à l'ouverture mise à profit. Dans la tâche meine_klasse, les élèves doivent s'identifier sur leur photo de groupe en se situant les uns par rapport aux autres. Cette tâche permet d'acquérir un certain nombre de moyens linguistiques (verbes de position) et de manipuler les prépositions spatiales sans avoir dans un premier temps à se soucier de la morphologie nominale, les marques casuelles ne s'appliquant pas aux prénoms. Ici aussi, l'information grammaticale apportée est d'un autre ordre: elle s'appuie sur une description conceptuelle qui s'avère être similaire dans les langues source et cible. En effet, en français comme en allemand, il y a mise en relation d'un relatum (point de repère) avec une entité (personne ou objet) par le biais d'une forme verbale (figure 2). Le fait que les schémas conceptuels se recouvrent offre la possibilité de faciliter le traitement des formes en L2.

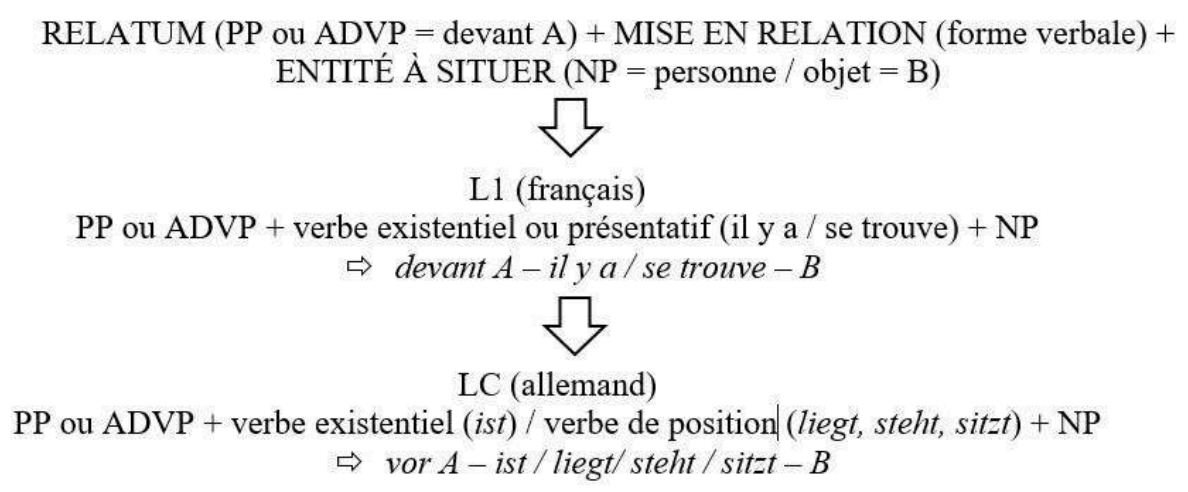

Figure 2 : Encodage de la localisation spatiale en L1 et L2 à partir d'un schéma conceptuel commun aux deux langues

Dans la réalisation de la tâche visant la description d'une pièce, il est frappant de constater que les réalisations de l'ouverture sont majoritairement correctes (graphique 2) et qu'un grand nombre d'élèves (18 sur les 21 ayant effectué la tâche) font usage de l'ouverture pour mettre en place le décalage spatial attendu. L'approche conceptuelle suggérée semble alléger le coût cognitif requis pour le traitement des énoncés à produire et promouvoir dans le même temps un positionnement verbal correct. 


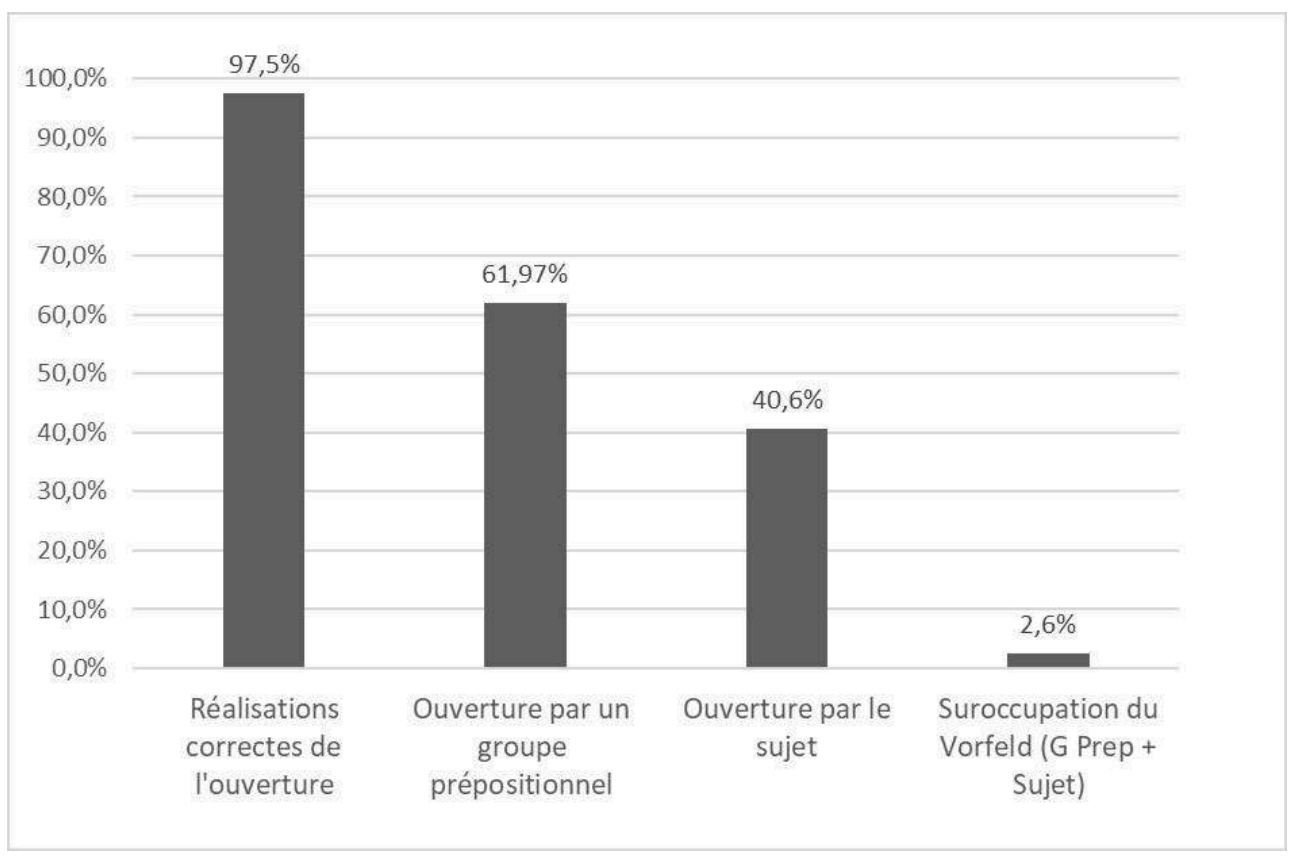

Graphique 2 : Réalisations de l'ouverture pour les énoncés relatifs à la description spatiale (tâche zimmer)

Quant à l'expression anaphorique $d a$, acquise en amont à travers des usages interactifs, puis réactivée pour le marquage chronologique, elle est chez plusieurs élèves reconfigurée pour son nouveau contexte d'emploi (32 occurrences sur les 145 expressions prépositionnelles utilisées à l'ouverture), avec une variété de formes inédites que l'on peut observer dans les exemples (7-10).

(7) (MAE_zimmer_routinen)

193. Gegenüber der (dem) Etagenbett steht einem (ein) Bücherregal. Darin ist (sind)

Bücher und Spiele.

[En face du lit superposé il y a une étagère. Dedans il y a mes livres et des jeux.]

(8) (JUL_zimmer_routinen)

149. Links neben mein (meinem) Bett steht eine Kommode und darauf ist ein(e) Lampe, (...)

[À gauche à côté de mon lit il y a une commode et dessus il y a une lampe, (...)]

(9) (PIE_zimmer_routinen)

319. (...) mein Zimmer sieht so aus: mein Bett ist auch in der Ecke, davor ist mein Schreibtisch, neben dem Schreibtisch ist das Fenster und links neben dem Fenster ist ein(e) Kommode, dahinter ist die Tür.

[(...) ma chambre ressemble à ça: mon lit est aussi dans le coin, devant il y a mon bureau, à côté du bureau, il y a la fenêtre et à gauche à côté de la fenêtre se trouve une commode, derrière c'est la porte.]

(10) (MEL_zimmer_routinen)

274. Auf dem Schreibtisch stehen eine Lampe, ein CD-Player und mein Computer. Darüber hängt ein kleines Bücherregal und darunter steht meine (mein) Papierkorb.

[Sur le bureau se trouvent une lampe, un lecteur CD et mon ordinateur. Au-dessus est suspendu une petite étagère et dessous il y a une corbeille à papier.]

\subsection{Vers une progression d'apprentissage}

41 Nous sommes consciente des limites de notre étude, menée dans un contexte précis auprès d'un unique groupe d'apprenants. Si les résultats obtenus ne sauraient être généralisés, du moins livrent-ils des pistes pour guider l'intervention et la manière de présenter les aspects formels de la langue. Le recours à des notions informationnelles 
et la volonté de dépasser le cadre de la phrase permettent d'avoir un nouvel éclairage sur des phénomènes langagiers réputés difficiles pour les apprenants (ici, le positionnement verbal second en allemand). Faire de l'appropriation de la fonction stratégique du champ initial un objectif permet d'envisager une progression susceptible de favoriser l'émergence de constructions plus conformes à l'usage, là où la règle positionnelle ne vise que la correction de la phrase.

La répétition de schémas interactifs basés sur la reprise topicale comme amorce de la réponse à une question permet de distinguer différentes valeurs informationnelles attribuées aux constituants de l'énoncé et d'aller au-delà de leur simple étiquetage catégoriel qui ne dit finalement pas grand-chose aux apprenants. Ces schémas permettent aussi de mieux saisir l'usage de certaines formes, comme l'expression plurifonctionnelle $d a$, qui s'avère très productive dans les domaines du temps et de l'espace. En focalisant les activités sur des réalisations adéquates de l'ouverture, nous avons pu constater que des associations Verbe-Sujet ont par ailleurs été mémorisées par de nombreux élèves à partir tout d'abord d'un nombre de verbes limité. Il semblerait que les apprenants puissent par la suite rappeler de telles associations et enrichir les combinaisons en intégrant une plus grande variété de bases verbales.

L'introduction des marqueurs de transition temporelle dann [ensuite] et danach [après ça] s'avère être facilitée par un usage fonctionnel précoce de $d a$. Nous faisons de cette étape un préalable à l'émergence et à l'usage d'autres expressions adverbiales pronominales à partir des prépositions spatiales : darauf, daneben, davor, darunter, ... [làdessus (sur ça), à côté (de ça), devant (ça), là-dessous (sous ça)], mais également la compréhension de leur rôle fonctionnel cohésif entre des énoncés successifs. À plus long terme, la maitrise de ces groupes prépositionnels peut soutenir l'insertion dans les énoncés de connecteurs argumentatifs construits à partir de cette même composante pronominale (da/des/dem) deshalb, deswegen, darum [c'est pour cela que] ou encore trotzdem, außerdem [malgré cela, en plus de ça] et qui jouent dans le champ initial la même fonction de raccord propositionnel.

1- Utilisation de l'expression anaphorique da pour ne pas répéter un moment, un lieu ou un domaine $>>2$ - Recours à dann comme marqueur de transition temporelle $>>3$ - Introduction de danach avec explicitation de sa fonction de raccord avec un contenu propositionnel activé $\gg$ 4- Apprentissage des prépositions spatiales (sans morphologie nominale) $>>5$ - Formation et manipulation de groupes adverbiaux pronominalisés pour la description spatiale $>>6$ - Vers l'appropriation des connecteurs discursifs

Figure 3 : Proposition d'une progression d'apprentissage relative aux marqueurs de cohésion en allemand L2

\section{En conclusion : quel terrain d'entente?}

Un des apports de l'approche actionnelle réside dans le rapprochement opéré entre usage et apprentissage d'une langue. Ce rapprochement nécessite d'articuler des objectifs linguistiques avec le développement de compétences communicatives taillées pour des usages. Le terrain d'entente se situe alors selon nous dans les perspectives que la didactique des langues est susceptible d'offrir aux praticiens en faisant en sorte que le savoir produit par les théories de référence (linguistique, recherche en acquisition, entre autres) leur soit accessible et pénètre les pratiques. Ces théories sont plurielles, elles mettent en relation les formes de la langue avec des fonctions spécifiques parfois 
mal connues (le rôle stratégique de l'ouverture en allemand) ; elles informent aussi sur les processus d'appropriation et sur la manière dont les apprenants peuvent traiter la L2 au fur et à mesure de leur progression. Il s'agit de s'interroger sur ce qui est apprenable ou enseignable à quel moment de cette progression.

Nous défendons l'idée que les enseignants doivent disposer pour cela d'une information grammaticale pertinente afin d'être en mesure d'enrichir les tâches d'une réflexion sur la langue qui ne se focalise pas uniquement sur la correction linguistique de phrases isolées, mais rende justice à l'utilisation effective de la langue dans des contextes variés. Nous rejoignons sur ce point les préconisations pour l'enseignement formulées par Dörnyei (2009 : 279-302), à savoir proposer des activités visant à manipuler du sens (c'est ce que permettent les tâches), recourir à l'apprentissage de séquences préfabriquées, soutenir l'automatisation de certaines procédures par des activités de pratique contrôlée conçues comme des étapes pour la réalisation de la tâche (forcer la reprise à l'ouverture par exemple) et, à l'instar de Kramsch, donner du sens aux formes en les mettant en relation avec leurs fonctions pragmatico-discursives.

\section{BIBLIOGRAPHIE}

BANGE, P., 2005, L'apprentissage d'une langue étrangère : Cognition et interaction, Paris, L'Harmattan.

BERTHOUD, A.-C.,1996, Paroles à propos : Approche énonciative et interactive du topic. Paris, Ophrys.

BOHNACKER, U., ROSÉN, C., 2008, "The clause-initial position in L2 German declaratives : Transfer of information structure", in Studies in Second Language Acquisition, Vol. 30, p. 511-538.

BRECKLE, M., ZINSMEISTER, H., 2010, "Starting a sentence in L2 German-Discourse annotation of a learner corpus", in Semantic approaches in natural language processing: Proceedings of the Conference on Natural Language Processing 2010, p. 181-185.

CARROLL, M., LAMBERT, M., 2003, « Information structure in narratives and the role of grammaticised knowledge ", in C. Dimroth, M. Starren (eds.), Information Structure and the Dynamics of Language Acquisition, John Benjamins, Amsterdam, p. 267-287.

CARROLL, M., LAMBERT, M., 2006, « Reorganizing Principles of Information Structure in Advanced L2s, in H. Byrnes, H. Weger-Guntharp, K. A. Sprang (eds.), Educating for Advanced Foreign Language Capacities. Constructs, Curriculum, Instruction, Assessment, Georgetown University Press, Georgetown, p. 54-73.

CONSEIL DE L'EUROPE, 2001, Cadre européen commun de référence pour les langues, Didier, Paris. DALMAS M., 2005, « Dringend nötig: der "Blick über den Satzrand”. Zu den Schwierigkeiten von französischen Studierenden bei der Texterzeugung ", dans K. Adamzik, W.-D. Krause (dirs), TextArbeiten. Textsorten im fremd-und muttersprachlichen Unterricht an Schule und Hochschule, Gunter Narr, Tübingen, p. 97-109. 
DALMAS M., 2013, « Strategien der Fokussierung: Eine "spannende" Geschichte - und eine Revidierung mancher Vorurteile », im S. Adam (dir), « Informationsstrukturen » im gesteuerten Spracherwerb. Französisch - Deutsch kontrastiv, Peter Lang, Frankfurt am Main, p. 67-77.

DÖRNYEI Z., 2009, The Psychology of Second Language Acquisition, Oxford University Press, Oxford, New York.

ELLIS, R., 2006, «Current Issues in the Teaching of Grammar : An SLA Perspective », TESOL Quarterly, 40(1), p. 83-107.

ELLIS, R., 2008, The study of second language acquisition, Oxford, New York, Oxford University Press.

FANDRYCH C., 2003, «Zur Textlinguistik des Vorfelds », im M. Thurmair, E.-M. Willkop (dirs), Am Anfang war der Text. 10 Jahre « Textgrammatik der deutschen Sprache », Iudicium Verlag, München, p. 173-196.

FANDRYCH C., 2005, « Ordnung und Variation in Satz und Text », Fremdsprache Deutsch, 32, p. 5-12.

FELCE, C., 2015, L'ouverture de l'énoncé en allemand L2 : De la compréhension d'un phénomène à son appropriation et à son enseignement. Perspectives en didactique des langues [Thèse de doctorat en sciences du langage, sous la direction de D. Macaire et de D. Véronique]. Paris 3 Sorbonne Nouvelle.

KRAMSCH C. J., 1983, "Discourse Function of Grammar Rules: Topic Construction in German", Modern Language Journal, 67, 1, p. 13-22.

LAMBERT M., CARROLL M., STUTTERHEIM C. von, 2008, « Acquisition en L2 des principes d'organisation de récits spécifiques aux langues ", Acquisition et interaction en langue étrangère, 26, p. 11-31.

LIPSKY A., 2010, « Vorfeldfehler in Texten von Deutschlernenden », Deutsch als Fremdsprache, 47. Jahrgang, Heft 2, p. 70-76.

NARCY-COMBES J.-P., 2005, Didactique des langues et TIC : vers une recherche-action responsable, Ophrys, Paris.

POCHON-BERGER, E., 2008, « La reprise comme ressource interactionnelle en langue seconde », Revue Tranel, 48, p. 43-61.

SAMSON G., 2004, « L'attaque de l'énoncé assertif en allemand », dans P. Cotte, M. Dalmas, H. Włodarczyk (éds), Énoncer : l'ordre informatif dans les langues, Paris, L’Harmattan, p. 185-206.

SCHANEN, F., CONFAIS, J.-P., 2005, Grammaire de l'allemand : Formes et fonctions, Paris, Armand Colin.

SKEHAN P., 1998, A cognitive approach to language learning, Oxford University Press, Oxford.

SKEHAN P., FOSTER P., 2001, "Cognition and Tasks", in P. Robinson (ed), Cognition and Second Language Instruction, Cambridge University Press, New York, p. 183-205.

STUTTERHEIM C. von, 1997, Einige Prinzipien des Textaufbaus: Empirische Untersuchungen zur Produktion mündlicher Texte, Niemeyer, Tübingen.

STUTTERHEIM C. von, NÜSE R., MURCIA-SERRA J., 2002, « Différences translinguistiques dans la conceptualisation des événements ", Revue française de linguistique appliquée, Vol. VII, 2, p. 99-115.

WATOREK, M., 2005, « Domaine de la spatialité : Construire une description spatiale en langue étrangère », Letras, 30/31, p. 169-184. 
WÖLLSTEIN A., 2010, Topologisches Satzmodell, Heidelberg, Universitätsverlag Winter.

ZIFONUN, G., HOFFMANN, L., STRECKER, B., BALLWEG, J., 1997, Grammatik der deutschen Sprache, Vol. 1-3, de Gruyter, Berlin, New-York.

\section{RÉSUMÉS}

Les règles de grammaire constituent des connaissances déclaratives qui font l'objet d'un enseignement explicite en classe de langue et dont les enseignants attendent bien souvent qu'elles soient directement opérationnelles dans la production en langue cible. Or, si elles décrivent des critères essentiels à la correction de la phrase, elles ne peuvent que rarement être mobilisées telles quelles dans les performances des apprenants. Dans cette contribution, nous présentons des pistes destinées à favoriser l'appropriation de la construction des énoncés assertifs en allemand L2. Dans le cadre de l'approche actionnelle, il nous semble en effet indispensable de concevoir la grammaire autrement qu'à travers des règles prescriptives et une terminologie abstraite, ignorant l'usage de la langue, le contexte discursif des énoncés et le travail cognitif des apprenants. Partant de tâches proposées en classe et de l'analyse de productions auxquelles elles ont donné lieu, il s'agit d'identifier les enjeux acquisitionnels qu'elles représentent et d'apporter aux apprenants une information grammaticale pertinente afin qu'ils puissent saisir (au sens de comprendre et s'approprier) le fonctionnement des énoncés dans leur contexte pragmatico-discursif.

Grammar rules are part of the declarative knowledge explicitly taught in the language classroom and they are expected to be directly applied by the L2 learner. However, although morphosyntactic rules describe essential parameters for the sentence correction, they cannot be used as such in the performance of learners, especially in real time communication. This paper aims to present findings about how beginner learners could be supported in their appropriation of the construction of assertive utterances in German L2. Within the task-based approach initiated by the CEFR, it seems necessary to consider grammar differently and not only as a set of rules or abstract categories, disconnected not only from usage and discursive context, but also from the cognitive aspects of learning. By identifying learning opportunities in classroom tasks and in analysing the associated performances, a progression can be sketched out. The aim is to provide learners with the relevant forms and grammatical information that will enable them to understand and acquire how to build utterances with regard to their pragmatic-discursive context.

\section{INDEX}

Mots-clés : allemand langue étrangère, approche à visée actionnelle, didactique des langues, enseignement secondaire, grammaire en contexte, syntaxe

Keywords : foreign language didactics, German as a Foreign Language, secondary school instruction, syntax, Task-based language teaching (TBLT), usage-based grammar

\section{AUTEUR}

\section{CATHERINE FELCE}

Université de Grenoble Alpes, Lidilem 